\title{
O Diagnóstico de Delirium
}

\author{
Afonso Carlos Neves*
}

\section{RESUMO}

O quadro de delirium, ou confusão mental aguda, é relativamente comum, principalmente entre pacientes geriátricos. No entanto, trata-se de comprometimento pouco estudado e controverso entre os autores. Com o DSM-IIIR passou a ser possivel uma uniformidade na avaliação desses quadros. Paraavaliaçāoquantitativado delirium pode ser útil o uso do "mini exame do estado mental".

\section{UNITERMOS}

Delirium. Diagnóstico. Confusāo mental.
- Doutor em Neurologia pela Escola Paulista de Medicina. Chefe do Setor de Urgências da Disciplina de Neurologia da Escola Paulista de Medicina.
O quadro de delirium, mais conhecido no meio médico e leigo como confusão mental aguda, é um comprometimento agudo do conteúdo da consciência. Assim sendo, temos necessariamente que nos remeter ao conceito de consciência. Segundo William James (citado por Sanvito, 1978), "todos sabem o que é consciência até que tentem definí-la". Trata-se de assunto de complexa abrangência, sendo abordado tanto por ciências humanas como biológicas. Em Medicina conceitua-se consciência como o estado de completo conhecimento de si mesmo e do meio ambiente (Sanvito, 1978; Plum \& Posner, 1987).

A alteração da consciência pode ocorrer de forma variada, podendo ser enquadrada em diversas categorias. $\mathrm{O}$ comprometimento limitado de determinadas funções superiores, como a afasia, por exemplo, reduz o conteúdo total da consciência; no entanto, esse tipo de distúrbio, com dano cerebral localizado, não é considerado como estado alterado de consciência. De modo geral, pode-se agrupar os estados alterados de consciência em dois grandes grupos: os estados em que haja, predominantemente, um comprometimento de nível de consciência, e os estados em que haja, predominantemente, um comprometimento de conteúdo da consciência. No primeiro grupo estão os comas em seus diversos graus; no segundo grupo estão os estados confusionais, também chamados pela literatura de delirium. Tanto o coma como o delirium têm, mais frequientemente, um curso agudo, sendo que este último dificilmente dura mais do que 4 a 7 dias, podendo eventualmente chegar a 30 dias. Os quadros subagudos ou crônicos compreendem, mais freqüientemente, a demência e os quadros vegetativos, entre outros (Plum \& Posner, 1987; Lipowski, 1987). Como este trabalho enfoca a confusão mental aguda ou delirium, nos deteremos mais sobre este quadro.

O primeiro médico, que se tenha conhecimento, que tenha utilizado o termo delirium, foi Celsus, no primeiro século antes de Cristo; Hipócrates utilizava o termo phrenitis para designar um quadro provavelmente semelhante (Lipowski, 1989).

O grande progresso no conhecimento dos distúrbios cognitivos adquiridos ocorreu a partir do século XIX, com descrições de neurossífilis, coréia de Huntington, doença de Pick. Em 1906, Alzheimer fez descriçōes histopatológicas de degeneração neuronal, observando placas senis no encéfalo de uma mulher com 55 anos e comprometimento progressivo de memória.

O vínculo entre cérebro e comportamento fora apenas suposto, mas a partir de Broca em 1865, com a descrição dos distúrbios de linguagem e lesão do hemisfério esquerdo, passou a ser assumido dentro dos padrōes de conhecimento científico moderno (Odenheimer, 1989). Por outro lado, a partir de Pinel em 1793, há uma progressão do enquadramento nosológico da síndrome confusional; esse autor considerou algumas das características desse processo na categoria do "idiotismo". Esquirol descreveu suas manifestações como "idiotia aguda". Georget e Delasiany (meados do século 19) definiram esse quadro como "estupidez adquirida", de modo semelhante aos psiquiatras alemães. Chaslin utilizou a denominação "afecção mental autônoma", denotando a semiologia e evolução de sua forma 
primitiva aguda e de suas variedades secundárias a outra patologia; foi ele quem primeiro utilizou o nome e descreveu a "síndrome confusional" em 1895. Entre os autores de língua inglesa, Hood, em 1870, relatou sete casos de delirium senil e concluiu que esse quadro poderia ocorrer em idosos sem "debilidade mental" prévia, podendo ser reversível com o tratamento de sua causa. Régis procurou dar uma unidade aos quadros confusionais como sendo a manifestação psicótica das intoxicações endógenas e exógenas. Marchand, Courtois e Toulouse consideraram as alterações confusionais graves como uma reação do sistema nervoso a diversas agressões, cujo substrato anátomo-patológico seria uma meningoencefalite difusa associada a lesōes inflamatórias e a lesões destrutivas. Desse modo, de 1793 a 1940, a síndrome confusional teve uma evolução de sua classificação dentro das psicoses agudas, com alteração de consciência freqüentemente reversível (Olivennes, 1989; Deniker et al., 1971; Lipowski, 1983). Embora, a partir de 1952, com o DSM-I (Diagnostic and Statistic Manual of Mental Disorders; $1^{\mathbf{a}}$ edição), tenha-se procurado sistematizar os critérios para diagnóstico dos quadros confusionais dentro da categoria delirium, atualmente ainda há divergências na terminologia e conceitos adotados.

Para conceituar delirium, nos valemos dos critérios estabelecidos pelo DSM-IIIR (Diagnostic and Statistical Manual of Mental Disorders; $3^{\mathbf{a}}$ edição revista; The American Psychiatric Association, 1987), que agora passamos a relatar:

A. Capacidade reduzida para manter a atenção a estímulos externos (por exemplo, as perguntas precisam ser repetidas devido à atenção que se dispersa) e para mudar adequadamente a atenção para novos estímulos externos (por exemplo, respostas perseverantes a uma pergunta prévia).

B. Pensamento desorganizado, indicado através de um discurso incoerente, irrelevante, divagante.

C. Pelo menos dois dos seguintes:

1. Redução do nível de consciência, por exemplo, dificuldade em se manter alerta durante o exame.

2. Distúrbios perceptivos: interpretaçöes falsas, ilusões e alucinações.

3. Perturbação do ciclo sono-viǵ́lia, com insônia ou sonolência durante o dia.

4. Aumento ou diminuição da atividade psicomotora.

5. Desorientação no tempo, espaço e quanto a si próprio.

6. Comprometimento da memoria, por exemplo, incapacidade de fixar coisas novas, tais como nomes de diversos objetos sem relação um com o outro após cinco minutos, ou de se lembrar de eventos passados, tais como a história do atual episódio de enfermidade.

D. Características clínicas que se desenvolvem num curto período de tempo (usualmente horas ou dias) e tendem a flutuar ao longo do dia.
E. Ou (1) ou (2) :

(1) - Evidência a partir da história, exame físico, ou testes laboratoriais de um específico fator orgânico (ou fatores) que se julgue estar etiologicamente relacionado com o distúrbio.

(2) - $\mathrm{Na}$ ausência de tal evidência, um fator orgânico etiol6gico pode ser presumido, se o distúrbio não puder ser atribuído a nenhum distúrbio mental não orgânico, por exemplo, episódio maníaco, responsável por agitaçāo e perturbação do sono.

Cada um desses itens pode estar presente em variados graus, o que requer certa atenção do observador. Em um quadio de delirium leve o comprometimento do curso do pensamento pode se manifestar apenas por discreta aceleração ou lentificação do pensamento. Em um delirium grave, com o pensamento bastante desorganizado, pode ser difícil, ou mesmo impossível a avaliação das funçōes como orientação e memória; são os quadros que costumam ser chamados de agitação psicomotora.

Quanto à orientação, em delirium de grau leve a desorientação temporal pode ser o primeiro sintoma. A desorientação quanto a si próprio é raramente observada. Perturbações emocionais associadas são comuns, o que pode induzir a erro diagnóstico, conduzindo a hipóteses psiquiátricas .

Não é comum, mas pode haver a presença de movimentos anormais, como várias formas de tremor, sinais de distúrbio do sistema nervoso autônomo, além de disnomia e disgrafia .

O delirium pode iniciar-se de forma abrupta ou ser precedido por horas ou dias de sintomas prodrômicos, que podem incluir inquietação, falta de clareza no pensamento, insônia noturna, hipersonolência diurna, pesadelos e hipersensibilidade a estímulos visuais e auditivos. A flutuação nos sintomas é ocorrência comum no delirium, sendo que os intervalos lúcidos são mais comuns pela manhã. História de comprometimento cerebral pregresso, ocorrência prévia de delirium, cérebro imaturo ou envelhecimento são fatores que tornam o indivíduo mais suscetível a apresentar quadro de delirium.

$\mathrm{Na}$ maioria das vezes o delirium dura até cerca de uma semana; dificilmente perdura por mais de um mês. Na medida em que se corrija o distúrbio de base, a recuperação pode ser completa. Se o distúrbio de base persistir, a síndrome do delirium pode evoluir para outra síndrome mental orgânica mais estável, ou mesmo chegar até a morte .

Com frequiência, delirium e demência podem coexistir, sendo que a presença do delirium dificulta o diagnóstico da demência; neste caso, somente a certeza de uma demência prévia permite os dois diagnósticos. Em caso de dúvida é mais adequado fazer a hipótese diagnóstica de delirium, o que leva a condutas mais ativas, não trazendo riscos ao paciente. De modo geral, é requerido um mínimo de 6 meses de comprometimento mental para firmar a hipótese de demência, enquanto que o delirium tem a duração citada acima. Assim, outros 
(Martin, 1987) preferem o termo "estado confusional subagudo" para os quadros que estejam entre 1 e 6 meses.

Quanto à compreensão do processo fisiopatológico do delirium, embora os fatores etiologicos sejam bastante variados, podem ser aventados alguns pontos em comum, no que diz respeito ao comprometimento neurológico. Por "funções superiores" entende-se as atividades cognitivas que são mais desenvolvidas no homem, embora alguns animais possam, aparentemente, ter alguma atividade desse tipo. Por atividades cognitivas entende-se como as que são responsáveis pelos processos envolvidos no aprendizado, compreendendo então o registro, o processamento e o armazenamento de informações. Sendo assim, a atenção tem importante papel no registro e codificação do material a ser armazenado. $O$ processamento inclui o pensamento e o raciocínio, através da comparação e estabelecimento das relações entre idéias e imagens. Sabe-se atualmente que não só o neocórtex é importante na regulação das funções superiores, mas também mecanismos subcorticais participam. $O$ circuito límbico contribui no processo da memória. O hipotálamo tem função em atividades emocionais, como a agressividade e sexualidade. Há conexões entre as atividades ligadas à emoção e às funções superiores (Vingoe, 1982). As emoções são admitidas como sendo estados produzidos por reforço de estímulos. Há evidências de que a amígdala esteja envolvida na formação das associações que reforçam os estímulos; o córtex orbitofrontal está correlacionado com a correção ou ajustamento das respostas comportamentais, de acordo com o reforço ambiental negativo ou positivo. Há ligações do córtex orbitofrontal e amígdala com o hipotálamo e com o striatum, além de outras regiões corticais (Rolls, 1986).

Por fim, tem papel no estudo de funçōes cognitivas e suas alterações, a interação cérebro-mente; assunto que tem sido bastante discutido atualmente, foi objeto de pesquisa de grandes estudiosos em Neurologia (Penfield, 1983; Eccles, 1977; Popper, \& Eccles, 1977; Vigotskii, Luria, \& Leontiev, 1988). Os diversos e renomados autores têm divergências quanto à maneira de compreender essa interação. Trata-se de assunto longe de ser esgotado, no qual, de nossa parte, $\epsilon$ importante ressalvar que não se reduza o psíquico ao orgânico, ou o orgânico ao psíquico, o que, em tratando-se de delirium e seus diagnósticos diferenciais, poderia limitar a abrangência diagnóstica diante de quadros dessa natureza.

Na avaliação clínica das síndromes mentais tem papel fundamental o exame do estado mental. São diversos os testes neuropsicológicos que podem ser utilizados. De modo geral, são relativamente extensos e demandam certo tempo para realização, bem como adestramento adequado do observador. Um teste de realização simples e que requer pouco tempo para execução, além de exigir um treino menor por parte do observador, é o "Mini Mental State Examination (MMSE)" (Folstein; Folstein; Mchugh, 1975). Trata-se de um teste simplificado que inclui onze questões e requer cinco a dez minutos para ser realizado. Essas questões estão concentradas em cinco itens que correspondem a: orientação, memória imediata, atenção e cálculo, memória de evocação, linguagem. Foi chamado "mini" por concentrar-se apenas em aspectos cognitivos das funçōes mentais, excluindo questões referentes a humor, experiências mentais anormais e a forma de pensamento; assim, ele separa pacientes com distúrbios cognitivos dos que não têm esse distúrbio. Evidentemente ele não substitui uma avaliação clínica completa para atingir um diagnóstico. No entanto, pode ser bastante útil em casos de urgência, como são os de delirium, que necessitam relativa rapidez na investigação de sua etiologia e nas primeiras medidas terapêuticas.

Em relação aos fatores etiológicos do delirium, as causas sistêmicas costumam estar entre as primeiras a serem aventadas, pela sua frequência, embora as causas neurológicas propriamente ditas sejam comuns. A real necessidade dessa investigação deve ser ponderada pelo sofrimento que pode causar a um paciente, talvez já debilitado e sistemicamente comprometido, além do ônus econômico que pode impor ao mesmo (Shaw et col., 1982).

O delirium é um distúrbio relativamente comum em pacientes idosos hospitalizados, embora seja o quadro menos investigado e compreendido nesses pacientes (Bayne, 1978; Berthaux, 1986; Lipowski, 1989), tendo talvez menor ocorrência em unidades de Pronto Socorro, onde pode ser mais difícil sua abordagem diagnóstica. As pesquisas sobre a epidemiologia, fisiopatologia, prevenção e tratamento desse quadro são escassas e algo controversas. Estima-se que sua incidência em pacientes hospitalizados seja em torno de 10 a $15 \%$, sendo que essa proporção aumenta em pacientes idosos hospitalizados para um terço a metade dos casos (Goldman, 1989).

\section{SUMMARY}

Delirium, also called acute confusional state, is not a rare disturb, mainly in geriatric pacients. Nevertheless, this condition is not very studied and it is controversial in literature. After DSM-IIIR it became possible a uniformity in the approch of delirium. For a quantitative examination it is usefull the Mini Mental State Examination.

\section{KEY WORDS}

Delirium, diagnosis, acute confusional state.

\section{Bibliografia}

1. AMERICAN PSYCHIATRIC ASSOCIATION - Manual de diagnóstico e Estatística de Distúrbios Mentais - Editora Manole Ltda., 1989, do original americano de 1987.

2. BAYNE, J.R.D. - Management of confusion in elderly persons. Canadian Medical Association Journal 118: 139-141, 1978.

3. BERTHAUX, P. - Les troubles de vigilance et les etats confusionnels du sujet âgé. Le point de vue de gériatre. Semaine des Hopitaux de Paris 62: 2801-2802, 1986.

4. DENIKER, P; COLONNA, L.; GAUSSEL, J.J. - Syndrome confusionnel. Etude semiologique. La Revue de Medecine 27: 1735 - 1738, 1971.

5. ECCLES, J.C. - O conhecimento do cérebro. Atheneu Editora, São Paulo, 1979.

6. FOLSTEIN, M.F.; FOLSTEIN, S.E.; MCHUGH, P.R. - "Mini mental state: A practical method for guiding the cognitive state of patients 
for the clinician. Journal of Psychiatric Research 12: 189-198, 1975.

7. GOLDMAN,L.S. in Psychiatry, Diagnosis \& Therapy. Flaherty, Channon \& Davis , Appleton \& Lange, 1989.

8. LIPOWSKI, Z.J. - Transient cognitive disorders (delirium, acute confusional states) in the elderly. American Journal of Psychiatry 140: 1426-1436, 1983.

9. LIPOWSKI, Z.J. - Delirium (acute confusional states). The Journal of the American Medical Association 258: 1789-1792, 1987.

10. LIPOWSKI, Z.J. - Delirium in older adults. Advances in Psychosomatic Medicine 19: 1 - 16, 1989.

11. MARTIN, F.C. - Diagnosis and management of acute confusional states. The Practitioner 231: 848 - 852, 1987.

12. ODENHEIMER, G.L. - Acquired cognitive disorders of the elderly. Medical Clinics of North America 73: 1383 - 1411, 1989.

13. OLIVENNES, A. - Propositions pour une nouvelle classification des syndromes confusionnels. Annales Medico-Psychologiques 147 $679-684,1989$.

14. PENFIELD, W. - O mistério da mente. Atheneu Editora. São Paulo, 1983.
15. PLUM, F. \& POSNER, J.B. - The dlagnosis of Stupor and Coma, Third Edition. F.A. Davis Company, Philadelphia, 1987

16. POPPER, K.R. \& ECCLES, J.C. - The self and its brain. SpringerVerlag, New York, 1977

17. ROLLS, E.T. - A theory of emotion, and its application to understunding the neural basis of emotion in Emotions, neuronal and chemical control, edited by Oomura, Y., published by Japan Cientific Societies Press, Tokyo, pp. 325-344, 1986.

18. SANVITO, W.L. - Os comas na prática médica. Editora Manole Ltda, São Paulo, 1978.

19. SHAW, D.M.; KELLAM, A.M.P. \& MOTTRAM,R.F. - Brain Sciences In Psychiatry. Butterworth \& Co Ltd, 1982.

20. VIGOTSKII, L.S.; LURIA, A.R.; LEONTIEV, A.N. - Linguagem, desenvolvimento $e$ aprendizagem. Editora da Universidade de Săo Paulo, 1988.

21. VINGOE, F.J. - Higher functions of the nervous system in Brain Sciences in Psychlatry, editado por Shaw, D.M., Kellam, A.M.P. \& Mottram, R.F. Butterworth \& Co LTd, London, pp.130-146, 1982. 